\title{
DIÁSTOLE ZERO OU REVERSA À DOPPLERVELOCIMETRIA DAS ARTÉRIAS UMBILLCAIS
}

*R.P.V. Francisco, R.M.Y. Nomura, S. Miyadahira, M. Zugaib

Setor de Vitalidade Fetal, Clínica Obstétrica - Hospital das Clínicas e Faculdade

de Medicina da Universidade de São Paulo

UniteRMOS: Diástole zero. Diástole reversa. Dopplervelocimetria. Artérias umbilicais. Ultra-sonografia.

KErwords: Absent end-diastolic velocity waveform. Reversed end-diastolic velocity waveform. Dopplervelocimetry. Umbilical arteries. Ultrasonography.

\section{INTRODUÇÃO}

Antes do advento da técnica dopplervelocimétrica, o diagnóstico dos transtornos da placentação baseava-se nas suas conseqüências, ou seja, nas ocorrências de crescimento inadequado do feto ou de distúrbios de sua oxigenação caracterizados pelo sofrimento fetal. Mudanças radicais tiveram lugar quando pioneiramente, em 1977, Fitzgerald; Drumm', combinando a técnica da ultra-sonografia com o dispositivo de doppler contínuo, desenvolveram um novo método que possibilitava o estudo do fluxo sangüíneo nas artérias e na veia umbilicais, de forma não-invasiva e segura. Subseqüentemente, muitas pesquisas foram levadas a cabo demonstrando que a dopplervelocimetria das artérias uterinas e umbilicais é muito útil no diagnóstico da insuficiência placentária identificando as gestações nas quais os fetos são submetidos a elevado risco de hipoxemia. Vislumbrava-se assim o uso desta nova técnica na avaliação qualita-

*Correspondência:

R. Passo da Pátria 855, 11 -BelaAliança-Cep: 05085-000 SãoPaulo-SP-Tel/Fax: (1 1)3641-8279

E-mail:rpvf@uol.com.br-HomePage:wmw.hcnet.usp.br/ob/ tiva da placenta precedendo os distúrbios no compartimento fetal.

Em face às possibilidades de se atestar em humanos o que já se sabia há muito tempo em animais por meio de experimentos laboratoriais, a dopplervelocimetria, uma técnica ainda inusitada e tida como promissora, mobilizou inúmeros pesquisadores que se dedicaram aos seus estudos experimentais em laboratórios com modelos animais e clínicos, desvendando todos os detalhes que se têm conhecimento atualmente. Por razões exclusivamente técnicas, os métodos qualitativos sobrepujaram os quantitativos prevalecendo na abordagem do produto conceptual, feto e placenta.

Como forma de interpretação dos sonogramas, à par dos índices criados, levando-se em conta a velocidade máxima sistólica, a velocidade mínima no final da diástole e a velocidade média em todo o ciclo cardíaco, desde logo, as formas da onda também chamaram a atenção de muitos pesquisadores. Desta maneira, em pouco tempo verificou-se que a ausência de fluxo na diástole (DZ) e, notadamente, o fluxo reverso (DR) são alterações doppler- velocimétricas muito graves, denotando importante grau de insuficiência placentária que se relaciona com altas taxas de mortalidade e morbidade perinatais ${ }^{2,3}$. Em virtude disso, a identificação destes casos possibilita melhor vigilância pela instituição de cuidados diferenciados por meio de propedêutica ampla e completa, objetivando a diminuição dos riscos de comprometimento perinatal mais grave $e^{4,5}$.

Embora se constatem, na literatura compulsada, numerosos trabalhos a respeito dessas alterações placentárias graves, muitas lacunas permanecem a desafiar o tirocínio do obstetra tanto em sua atuação assistencial como na qualidade de pesquisador no que concerne à resolução obstétrica, já que muitas novidades na área propedêutica foram inseridas no contexto da avaliação global dos fetos submetidos às restrições ao seu desenvolvimento como sói ocorrer nestes casos.

\section{Conceito}

A DZ e a DR caracterizam insuficiência placentária gravíssima.

Diástole Zero (DZ): sonogramas de ar- 
térias umbilicais que exibem ausência de velocidade de fluxo durante a diástole.

Diástole Reversa (DR): sonogramas de artérias umbilicais com presença de velocidade de fluxo reverso durante a diástole.

\section{INCIDÊNCIA}

A incidência de DZ ou de DR em gestações normais é extremamente baixa. Johnstone et $a$. avaliando 160 gestações sem complicações, não observaram nenhum caso de DZ ou DR ${ }^{16}$.

Avaliando população selecionada de gestantes de alto risco para cursar com insuficiência placentária, a incidência de DZ ou de DR tem sido relatada entre $1 \%$ e $34 \% 11,17,18,19$. Esta alta variação da incidência pode ser justificada pelas diferenças das amostras populacionais de cada estudo. Considera-se aceitável incidência entre I e $4 \%$ quando se observam grupos de pacientes com várias doenças que participam da etiologia da insuficiência placentária ${ }^{20}$.

No Setor de Vitalidade Fetal da Clínica Obstétrica da FMUSP, no período de janeiro 1992 a junho de 1999, foram avaliadas 6251 gestações de alto risco, e entre estas, 225 casos de DZ ou de DR foram diagnosticados a partir da 20a semana de gestação, resultando em incidência de 3,6\%2! .

\section{Idade Gestacional no Momento do Diagnóstico de DZ}

\section{Primeiro trimestre}

A observação de DZ é achado normal no primeiro trimestre, sendo que a partir da $10^{a}$ semana de idade gestacional já se pode observar fluxo diastólico positivo em alguns casos e a partir da I 4a semana este deve estar presente em todos os $\operatorname{casos}^{29}$. É importante ressaltar que a verificação da DZ no primeiro trimestre não se associa a nenhum tipo de anormalidade ou doença fetal.

\section{Segundo e terceiro trimestres}

A observação de DZ no segundo e terceiro trimestres de gestação constituem importante fator preditor de resultado perinatal adverso. Fato importante e enfatizado por vários autores é a observação de que quanto mais precoce for a verificação da DZ, pior é o prognóstico desta gestação. Arabin et al. observaram que quando 0 diagnóstico de DZ se faz antes da $32^{\mathrm{a}}$ semana de idade gestacional, a mortalidade perinatal é mais elevada que nos casos em que a DZ foi verificada mais tardiamente ${ }^{12}$.

\section{Aspectos Técnicos}

O sonograma da artéria umbilical pode ser obtido em alça livre de cordão, e, nos casos em que são observadas velocidades diastólicas reduzidas, procura-se insonar a artéria umbilical na porção próxima à inserção placentária. A ausência de fluxo diastólico final na dopplervelocimetria das artérias umbilicais analisada próxima à inserção placentária caracteriza o diagnóstico de DZ, e a ocorrência de fluxo reverso neste local, durante a diástole, caracteriza a DR. A escoIha deste local para confirmar o diagnóstico de DZ ou DR visa minimizar os efeitos da variabilidade do comprimento e espessura do cordão umbilical sobre a resistência vascular $^{15}$.

Outro cuidado essencial é a manutenção do filtro de janela inferior em $50 \mathrm{~Hz}$, evitando-se que a detecção de DZ ou de DR possa ser superestimada.

\section{Fisiopatologia da Lesão Placentária}

Único órgão responsável pelo desenvolvimento fetal, a placenta, no final da gestação, exibe grande superfície de trocas da gestante com o feto, em média, de II metros quadrados, quando isenta de quaisquer anormalidades. Várias condições mórbidas maternas e gestacionais estreitam as possibilidades de fornecimento dos subs- tratos nutritivos e gasosos ao feto por interferirem com o processo de placentogênese, fenômeno de $1^{\circ}$ e $2^{\circ}$ trimestres, ou provocando, no $3^{\circ}$ trimestre, alterações vasculares nos troncos das vilosidades coriais, caracterizando a denominada insuficiência placentária.

Modelos experimentais podem ser utilizados para melhor demonstrar a fisiopatologia das lesões placentárias, principalmente quando se deseja abordar os aspectos hemodinâmicos. Nestes modelos pode-se, no final da gestação, embolizar vasos de troncos vilosos da placenta com microesferas, promovendo insuficiência funcional do órgão em graus variáveis, podendo atingir gravidade de intensidade suficiente para causar hipoxemia fetal progressiva. Do ponto de vista hemodinâmico, a diminuição de parênquima placentário funcionante restringe também a malha de arteríolas levando a um colapso no território vascular e aumento da resistência vascular placentária. Este evento pode ser estudado pela dopplervelocimetria, que inicialmente demonstra diminuiç̧ão da velocidade do fluxo sangüíneo durante a diástole culminando com fluxo diastólico ausente (DZ) ou reverso (DR) nas artérias umbilicais,7. Utilizando-se deste modelo, Morrow et al. (1989) demonstraram que a embolização progressiva de vasos placentários em ovelhas prenhes resulta na ausência de fluxo na diástole e até mesmo DR no sonograma das $A U^{8}$.

Estes experimentos simulam a situação clínica de insuficiência placentária que usualmente se caracteriza pela resistência vascular placentária elevada conferindo ao sonograma das AU baixa velocidade diastólica ${ }^{9}$, a qual associa-se à hipoxemia fetal, ao crescimento intra-uterino retardado e à morbidade perinatal elevada 10,11 .

O estudo anatomopatológico de casos que apresentam diástole zero ou reversa demonstra que raramente as placentas são normais. Arabin et al. ${ }^{12}$ encontraram sinais 
de insuficiência placentária em $74 \%$ dos casos. Miyadahira et d. ${ }^{13}$ observaram em 90 casos de DZ ou DR a presença de infartos vilosos em $50 \%$ dos casos. Os estudos clínicos e experimentais, aliados à avaliação anatomopatológica das placentas de casos em que se constatou a presença de DZ ou $D R$, permitem que se considere estes achados como sendo indicadores de insuficiência placentária ${ }^{7,9,14}$. Assim, fetos destas gestações estarão expostos às conseqüências desta situação clínica que, caracteristicamente, se traduzem em déficit nutricional e de oxigenação.

\section{Resposta Fetal à Hipoxia e Avaliação da Vitalidade Fetal}

A dopplervelocimetria pode ser utilizada para avaliar a resposta circulatória fetal à hipoxia por meio do perfil hemodinâmico fetal o qual, por ser exame não-invasivo e inócuo, tornou possível demonstrar em humanos o que se sabia em teoria por meio de experimentos laboratoriais em animais. Desta forma, os eventos hemodinâmicos decorrentes da resposta à hipoxia apresentada pelos fetos de gestações com fluxo anormal nas artérias umbilicais puderam ser amplamente estudados.

Estudos experimentais da década de 70 já haviam tornado claro que frente à hipoxemia observa-se a diminuição do fluxo sangüíneo para rins e membros com o intuito de proteger áreas mais nobres como coração, cérebro e supra-renais, um fenômeno denominado de centralização da circulação fetal. Simultaneamente, no território venoso descreve-se ainda fluxo preferencial pelo ducto venoso também com o objetivo de favorecer a oxigenação daquelas áreas ${ }^{31}$.

A dopplervelocimetria da circulação fetal, por possibilitar a monitorização destas alterações hemodinâmicas decorrentes da hipoxia, é considerada também um método de avaliação do bem-estar fetal. Assim, no

\section{Figura I - Probabilidade de acidose no nascimento de acordo com o índice de pulsatilidade para veias (IPV) do ducto venoso (DV) em casos de Diástole Zero ou reversa à dopplervelocimetria das artérias umbilicais. (Francisco, 1998)}

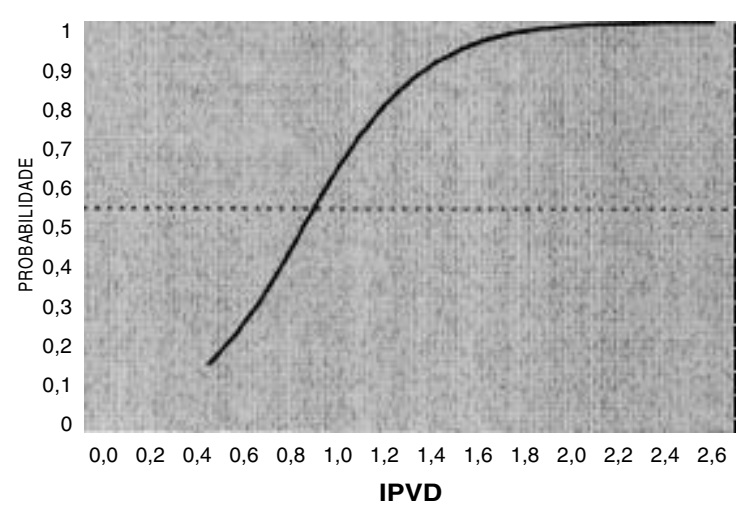

território arterial a diminuição do fluxo para rins e membros pode ser monitorizada pelo estudo da artéria aorta torácica descendente onde, devido a vasoconstricção ocorre decréscimo de seu fluxo durante a diástole. Ainda pode ser observada vasodilatação da artéria cerebral média que reflete no sonograma deste vaso pelo aumento do fluxo sangüíneo durante a diástole, visando proteger o sistema nervoso central dos agravos da hipoxia.

Após ser instalada vasoconstricção periférica intensa, ocorre aumento da resistência neste território e, conseqüentemente, aumento da pressão diastólica final no ventrículo direito e, com isso, estase na circulação de retorno às câmaras cardíacas. A seqüência de alterações hemodinâmicas fetais frente à hipoxia, descrita em estudos experimentais, passou assim a ser avaliada em fetos humanos com o advento da dopplervelocimetria. Observa-se que após a constatação de dopplervelocimetria anormal nas artérias umbilicais, ocorrem alterações na aorta torácica descendente (ATD), caracterizadas pelo aumento do índice de pulsatilidade (IP), seguidas pela vasodilatação da artéria cerebral média (diminuição do IP) e pela diminuição do fluxo sangüíneo no ducto venoso durante a contração atrial (aumento do IPV).

Enquanto a centralização mantiver compensada a oxigenação do sistema nervoso central, as atividades biofísicas permanecem inalterados. Com o agravamento da hipoxia observam-se alterações na veia cava inferior e no ducto venoso, ocorrendo diminuição do fluxo durante a contração atrial. Nos casos graves pode ser observado fluxo reverso no sonograma do ducto venoso durante a contração atrial. No sonograma da veia umbilical pode-se ainda notar a presença de pulsações.

A exaustão dos mecanismos compensatórios revela-se com os sinais de sofrimento fetal agudo, observando-se alterações importantes na cardiotocografia e no perfil biofísico fetal. Os resultados dopplervelocimétricos do ducto venoso se relacionam à ocorrência de acidose no nascimento, um reflexo do déficit respiratório placentário levando a comprometimento crônico da oxigenação do feto e, com isso, metabolismo anaeróbico fetal. Esta relação significativa permitiu a confecção de curva de probabilidade de acidose no nascimento de acordo com os valores do índice de pulsatilidade para veias (IPV) do ducto venoso (Figura I).

Na ausência da resolução da gestação, os casos de DZ ou de DR evoluem invaria- 
velmente para o sofrimento ou óbito fetal ${ }^{20}$. Casos que evoluíram para o óbito fetal apresentaram previamente anormalidades na redistribuição do fluxo sangüíneo nos territórios arterial e venoso fetal.

\section{INTERCORRÊNCIAS CLínICAS Associadas À DZ E DR}

\section{Hipertensão}

As síndromes hipertensivas comparecem com maior freqüência como doença materna participante da etiologia da insuficiência placentária grave resultando em DZ ou DR $3,12,23,33,43$. Farine et al. (1995), avaliando 16 estudos encontraram esta intercorrência clínica em 300 (57\%) das 785 pacientes avaliadas ${ }^{3}$.

Francisco (1998)22 observou síndromes hipertensivas presentes em $60 \%$ dos 30 casos de DZ ou DR, semelhante ao que observam Battaglia et al. (1993) ${ }^{23}$, que relataram estas síndromes em $65 \%$ das gestantes e Bell et al. (1992)24, que as referiram em $56 \%$ dos casos de DZ ou DR.

\section{Colagenoses}

Francisco (1998)22 constatou que a segunda doença mais freqüentemente encontrada em casos de DZ ou de DR foram as colagenoses, nas quais a presença de vasculopatias pode levar a insuficiência placentária ${ }^{25}$. Acrescente-se, ainda, que esta elevada ocorrência pode ser justificada por ser o HCFMUSP serviço de referência a portadores destas doenças, inclusive quando gestantes. Malcolm et al. ( 1991$)^{26}$ também referiram as colagenoses como a segunda doença materna mais freqüente, aparecendo em 12\% dos seus casos.

\section{Restrição do crescimento intra-uterino}

Em casos de DZ ou DR, a presença de insuficiência placentária, com alteração na nutrição fetal, é confirmada pelos trabalhos disponíveis na literatura médica, que constatam alta porcentagem de RN classificados

Figura 2 - Probabilidade de óbito de acordo com o peso fetal em casos de diástole zero ou reversa à dopplervelocimetria das artérias umbilicais. (Yamamoto etal., 2000)

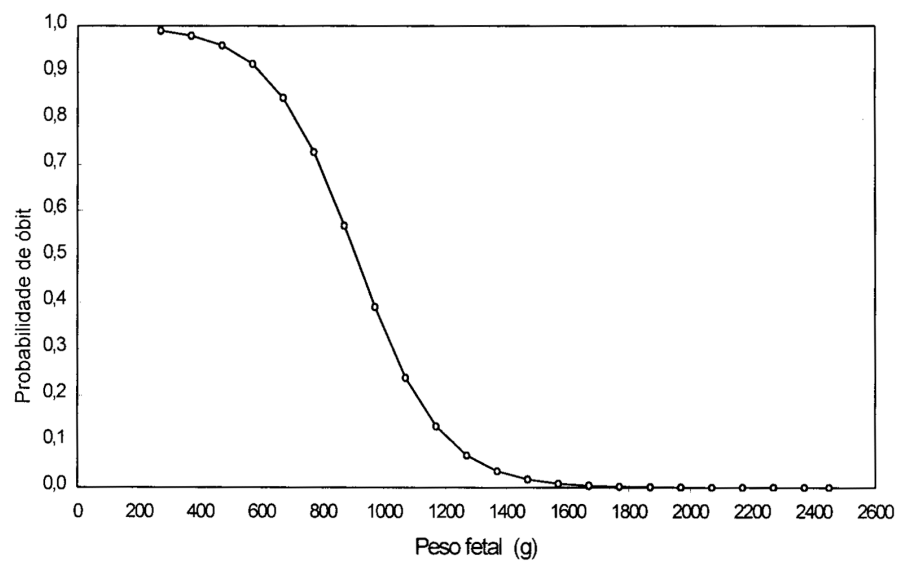

como PIG 3 , II, 12,24 (ver peso no nascimento).

\section{Malformações fetais}

A associação entre a DZ ou DR e as malformações e/ou cromossomopatias fetais tem sido descrita por vários autores²,11,24,27,28 aparecendo com taxas de 3 a 30\%.

\section{Resultados Neonatals}

Quanto aos resultados neonatais, nas gestações que cursam com DZ ou DR podem ser observadas altas taxas de índices de Apgar $<7$ tanto no $1^{\circ}$ ou $5^{\circ}$ minuto, incidência elevada de RN classificados como pequenos para idade gestacional (PIG), tempo prolongado de internação dos RN em Unidades Neonatais de Terapia Intensiva, freqüência elevada de óbitos fetais e neonatais ou seja, taxas de morbidade e mortalidade neonatais muito elevadas ${ }^{17,23,25}$.

\section{Idade gestacional no nascimento}

A média da idade gestacional no momento do parto varia de 29 a 33 semanas. Ressalte-se que, apesar das diferentes médias observadas nos diversos trabalhos, altos índices de prematuridade foram notados por todos autores. Battaglia et al. (1993)23 e KurkinenRäty et al. (1997) 31 , que descreveram média de 29, I e 31,7 semanas, respectivamente.
Rochelson (1989) ${ }^{25}$ e Weiss et al. (1992) ${ }^{32}$ notaram valores maiores do que a referida média, a saber, 33,9 e 32,4 semanas.

\section{Peso no nascimento}

A média do peso dos RN de acordo com dados da literatura médica revelam peso no nascimento variando entre $908 \mathrm{~g} \mathrm{e}$ |58| g, em casos de DZ ou DR $23,25,31,33$. Mcparland et al. (1990), que referiram peso superior a $1500 \mathrm{~g}$ somente em dois dos 29 RN avaliados ${ }^{34}$. Este grande comprometimento no peso de nascimento ilustra a gravidade da insuficiência placentária que caracterizam estas gestações.

Ainda em relação ao peso, alta porcentagem de RN são classificados como pequenos para idade gestacional variando de $60 \mathrm{a}$ $100 \%$ dos casos ${ }^{12,24,31}$.

Estudo realizado no HCFMUSP revelou que o risco para o óbito perinatal relacionouse muito ao peso do recém-nascido, principalmente para aqueles com peso inferior a l000g ou abaixo da 3 I $^{\mathrm{a}}$ semana de gestação, permitindo a elaboração de uma curva de probabilidade para o óbito perinatal de acordo com o peso do RN (figura 2), facilitando a interpretação do risco de óbito de acordo com o peso estimado do feto, nas gestações que cursam com DZ ou DR ${ }^{2 !}$. 


\section{Índice de Apgar no $\mathrm{I}^{\circ}$ minuto}

A ocorrência de índices de Apgar inferiores a 7 no $1^{\circ}$ minuto de vida tem sido relatada de 39 a $77 \%$ e, no $5^{\circ}$ minuto, de 9 a 46\%, em gestações com DZ ou DR ${ }^{12,24,31}$.

\section{Acidose no nascimento}

Na maioria das vezes, os fetos das gestações com DZ ou DR apresentam hipoxemia por diminuição da superfície de troca entre o binômio materno-fetal. Contrariamente, a ocorrência de acidose é muito variável. Enquanto Weiner (1990)35 não verificou acidose em gestações com DZ ou DR submetidas à cordocentese, apesar de diagnosticar hipoxemia em todos os fetos avaliados, outros autores referem que a acidose tem sido observada em aproximadamente $50 \%$ das gestações com DZ $Z^{12,17}$. No Brasil, Steinman et al. (1997) constataram acidose no nascimento $(\mathrm{pH}<7,20)$ em $50,8 \%$ dos 61 casos de DZ ou DR ${ }^{36}$, resultado semelhante ao encontrado por Francisco (1998) no qual 50\% das amostras de sangue de artéria umbilical revelaram acidose no nascimento ${ }^{22}$.

\section{Mortalidade Perinatal}

A mortalidade perinatal relacionada com a DZ ou DR revela-se entre 23 e 100\%, com grande variação pela inclusão ou não de fetos com malformações 3 , 17,37.38.

Lopes et al. (1990), em pesquisa realizada na Clínica Obstétrica do Hospital das Clínicas da Faculdade de Medicina da Universidade de São Paulo (HCFMUSP), observaram altos índices de mortalidade (80\%) em gestações com DZ, o que demonstra a gravidade destes casos, porém 23\% dos fetos estudados exibem malformações $^{39}$. Posteriormente, neste mesmo Serviço, Miyadahira et al. (1997a), estudando gestações em que se detecta DZ ou DR, observaram óbito perinatal em 49 dos 143 (34,26\%) casos e o óbito neonatal em 30
(20,98\%) casos. A mortalidade perinatal na DZ foi de 27,4\% (43 casos) e na DR de $63,8 \%(30 \text { casos })^{37}$. Por estes dados, podese perceber que a DR está relacionada a pior resultado perinatal, com mortalidade e morbidade perinatais extremamente elevadas e, por isso, conduta mais agressiva pode ser recomendada.

\section{Conduta nos Casos de DZ ou de DR}

Woo et al. (1987), estudando nove casos de DZ ou DR, observaram a evolução de cinco gestações para óbito intra-uterino, três, para óbito neonatal precoce, sobrevivendo apenas um RN. Neste estudo, os autores relacionaram a sobrevivência deste RN à interrupção imediata da gestação $0^{40}$. Esta conduta levou a aumento das taxas de prematuridade e, portanto, das complicações associadas a ela. Com objetivo de se diminuir a morte intra-uterina causada pela hipoxemia, decorrente da insuficiência placentária, provocou-se aumento da morbidade e da mortalidade neonatais pelas complicações associadas à prematuridade iatrogênica ${ }^{4 !}$.

Schulman (1987) associa a DZ a uma situação de extrema gravidade em que a vigilância do bem-estar fetal é obrigatória, devendo ser realizada em curtos intervalos de tempo ${ }^{41}$. Pillai; James (1990) concordam com estas observações e ressaltam, ainda, que o número de dias entre a detecção de DZ ou DR e o diagnóstico de sofrimento fetal, baseado em CTG e PBF, não é passível de prévia determinação, atingindo 9 semanas em seu estudo ${ }^{4}$.

Bekedam et al. (1990) descreveram cinco gestações com DZ acompanhadas por mais de três semanas até a necessidade de resolução obstétrica. Relatam a interrupção de uma das gestações na $3 \mathrm{I}^{\mathrm{a}}$ semana, ao fim de sete semanas de convivência do feto com a DZ ${ }^{43}$. Referem, portanto, que o diagnóstico de DZ não coincide necessari- amente com o momento em que se deve interromper a gestação. Particularmente, no caso descrito, a conduta médica adotada possibilitou o nascimento de RN viável, sujeito a morbidade menor.

Farine et al. $(1993)^{3}$ defendem a posição obstétrica mais difundida, desde antes utilizada na Clínica Obstétrica do HCFMUSP21,37, que consiste na vigilância intensiva (diária) dos casos com DZ ou DR, com o objetivo de se evitarem mortes intrauterinas e de se diminuírem a morbidade e mortalidade neonatais. Esta abordagem clínica possibilita o acompanhamento de gestações com DZ ou DR e a conseqüente diminuição da prematuridade iatrogênica. Por outro lado, por envolver múltiplas variáveis, maternas e fetais, leva ao questionamento do melhor momento para a interrupção da gestação, pois a meta central é antecipar-se às lesões fetais irreversíveis provocadas pela hipoxia grave e evitar-se a iatrogenia decorrente da interrupção intempestiva e prematura.

Na Clínica Obstétrica do Hospital das Clínicas da FMUSP, diante de gestantes com DZ, preconiza-se a orientação demonstrada no organograma da figura 3.

I) Internação da paciente após o diagnóstico para repouso, controle clínico, realização de ultra-sonografia morfológica e avaliação rigorosa da vitalidade fetal. Na presença de anomalias fetais, a conduta estará vinculada a este diagnóstico;

2) Entre 20 e 26 semanas de idade gestacional, a dopplervelocimetria e o índice do líquido amniótico (ILA) são realizados 2 a 3 vezes por semana. A partir de 26 semanas realizamos cardiotocografia (CTG),perfil biofísico fetal (PBF), ILA e o perfil hemodinâmico fetal diariamente. A resolução da gestação é considerada possível nos casos em que a idade gestacional for superior a 26 semanas e 0 peso fetal estimado superior a $500 \mathrm{~g}$.

3) A partir de 28 semanas de gestação, quando a avaliação da vitalidade fetal se 
apresentar alterada, caracterizada pela ocorrência de desacelerações tardias na CTG ou PBF inferior a 6 ou ILA inferior a 3,0, é indicada resolução da gestação. Em situações de vitalidade fetal preservada, é indicada a avaliação da maturidade fetal através da análise do LA colhido por amniocentese. Quando as provas indicarem maturidade plena ou intermediária (Teste de Clements positivo no mínimo até o segundo tubo e índice citolipídico superior a 5\%), é optado pela resolução da gestação. Nos fetos imaturos, preconiza-se o repouso da paciente e a vigilância fetal intensiva, através da realização diária da cardiotocografia, por no mínimo 30 minutos, acompanhado da avaliação do PBF e do ILA. O perfil hemodinâmico fetal é realizado com intervalos de 24 a 72 horas e a maturidade é reavaliada em 7 a 14 dias. Quando apresentar DR ou alteração importante no IPV do DV (valor acima de 1,0 ), deve-se discutir o uso de corticosteróides e resolução da gestação.

4) A conduta resolutiva é adotada em doenças maternas graves, de difícil controle clínico ou também em gestações acima de 34 semanas.

\section{Conclusões}

Determinar o melhor momento para a resolução da gestação é o maior desafio após o diagnóstico da DZ ou DR. Esta decisão é particularmente crítica no final do segundo e início do terceiro trimestres da gestação, e geralmente se embasam em protocolos assistenciais (figura 3), porém condutas personalizadas são freqüentes quando são consideradas todas as informações obtidas a respeito da condição fetal e do quadro clínico materno.

O seguimento longitudinal cuidadoso dos casos com DZ ou DR permite acompanhar par e passo os mecanismos de adaptação da circulação fetal frente à hipoxemia, obtendo-se os melhores subsídios para a

\section{Figura 3-Protocolo de conduta na diástole zero ena diástole reversa nas artérias umbilicais.}

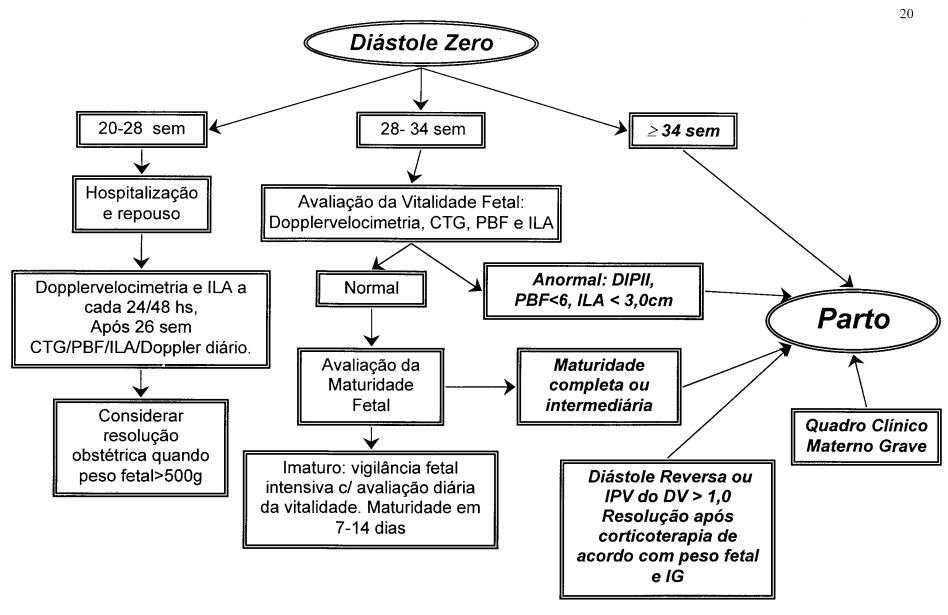

tomada de decisão. Por exemplo, a evolução da DZ para DR, e o progressivo aumento do índice de pulsatilidade da artéria umbilical, sinalizam comprometimento progressivo da circulação placentária. De outro lado, não obstante a sua importância, o diagnóstico de centralização da circulação, diagnosticada pela insonação da artéria cerebral média de forma isolada, não significa obrigatoriamente piora da vitalidade fetal, pois freqüentemente, nestas situações, o feto mantém as atividades biofíicicas adequadas as quais são representativas de boa oxigenação do sistema nervoso central.

Contudo, a partir dos resultados dopplervelocimétricos do sistema venoso fetal (valor do IPV do DV) pode-se estimar a probabilidade de ocorrência de acidose no nascimento o que dá maior clareza quanto a conduta obstétrica a ser tomada (figura I).

Além de se considerar prioritariamente 0 quadro clínico materno, a indicação da resolução da gestação deve ser pautada na propedêutica fetal que incluia biometria fetal (figura 2), as provas de maturidade fetal e todo o contingente de exames biofísicos destinados a avaliar o seu bem-estar, particularmente quando estes indiquem elevada probabilidade para acidose no nascimento (figura 3).

Portanto, nos casos de DZ ou DR é imprescindível rigorosa monitorização da vitalidade fetal através de todos os exames disponíveis visando evitar o diagnóstico tardio de sofrimento fetal. Em alguns casos o controle do bem-estar fetal permite que fetos extremamente prematuros e até mesmo inviáveis possam ser acompanhados por períodos prolongados, permitindo o nascimento de recém-nascido viável e em meIhores condições de maturidade. Neste aspecto, o perfil hemodinâmico fetal representa um papel fundamental, pois pode nos fornecer valiosas informações com respeito ao prognóstico da gestação, auxiliando na determinação do momento mais oportuno de resolução das gestações com DZ ou DR. Portanto, todos os esforços devem convergir para se evitar a interrupção iatrogênica com os grandes riscos da prematuridade "per se" e também evitar a interrupção tardia com os danos neurológicos irrever-

\section{Referências Biblográficas}

I. Fitzgerald DE, DrummJE. Non-invasive measurement of human fetal circulation using ultrasound: a new method. B. M. J., v.2, p. $1450-$ I, 1977.

2. Rochelson B, Schulman H, Farmakides G, Bracero L, Ducey J. Fleischer A, penny B, Winter D. The significance of absent-end diastolic velocity waveforms. Am J Obstet Gynecol 1987; | 56: | 213-8. síveis decorrentes de hipoxia prolongada. 
3. Farine D, Kelly EN, Ryan G, Morrow RJ, KnoxRitchie JW. Absent and reversed umbilical artery end-diastolic velocity. In Copel J, Reed KL, editores. Doppler ultrasound in obstetrics and gynecology. New York: Raven Press Ltd; 1995; p. 187-97.

4. Pillai M, James D. Continuation of normal neurobehavioural development in fetuses with absent umbilical arterial end diastolic velocities. Br. J. Obstet. Gynaecol., 1990,98:277-81.

5. Tyrrell SN, Lilford, R.J.; Macdonald, H.N. etal. Randomized comparison of routine vs highly selective use of Doppler ultrasound and biophysical scoring to investigate high risk pregnancies. Br. J. Obstet. Gynaecol., 1990,97: 909-16.

6. Trudinger BJ, Giles WB. Clinical and pathological correlation of umbilical and uterine artery waveforms. Clin. Obstet. Gynecol., 1989:32:669-78.

7. Gagnon R, Johnston L, Murotsuki J. Fetal placental embolization in late-gestation ovine fetus: Alterations in umbilical blood flow and fetal heart rate patterns. Am. J. Obstet Gynecol., 1996, 175:63-72.

8. Morrow R.J, Adamson SL, Bull SB, Ritchie JWK. Effect of placental embolization on the umbilical artery velocity waveforms in fetal sheep. Am. J. Obstet Gynecol., 1989, 161: 1055-64.

9. Blanco CE, Dawes GS, Hanson MA, Mccooke HB. Carotid baroreceptors in fetal and newborn sheep. Pedriatr. Res., 1988,24:342-346.

10. Ferrazzi E, Pardi G, Buscaglia M, et al. The correlation of biochemical monitoring versus umbilical flow velocity measurements of the human fetus. Am. J. Obstet. Gynecol., 1988, I 59: 1080-7.

I I. Trudinger BJ, Cook CM, Giles WB, et al. Fetal umbilical artery velocity waveforms and subsequent neonatal outcome. Br. J. Obstet. Gyanaecol., 199|,98:378-84.

12. Arabin B, Siebert M, Jimenez E, Saling E. Obstetrical characteristics of a loss end-diastolic velocities in the fetal aorta and/or umbilical artery using Doppler Gynecol. O bstet. Invest. , 1988,25: 173-80.

13. Miyadahira S, Yamamoto RM, Francisco RPV, Steinman DS, Schultz R, Zugaib M. Placental characteristics in pregnancies with absent or reversed end-diastolic velocity flow in the umbilical artey. Ultrasound Obstet Gynecol 1998, 12:159.

14. Trudinger BJ, Stevens D, Connely A, et al. Umbilical artery flow velocity waveforms and placental resistence: The effects of embolization of the umbilical circulations. Am. J. Obstet. Gynecol., 1987, I 57: |443-8.

15. Abramowicz JS, Warsof SL, Arrington J, Levy DL. Doppler analysis of the umbilical artery. The importance of choosing the placental end of the cord. J. Ultrasound Med. 1989,8:.219-21.

16. Johnstone FD, Haddad NG, Hoskins $P$ et al. Umbilical artery Doppler flow velocity waveform: The outcome of pregnancies with absent end-diastolic flow. Eur. J. Obstet. Gynecol. Reprod. Biol., |988,28:17|-8.
17. Brar HS, Platt LD. Reverse end diastolic flow velocity on umbilical artery velocimetry in high risk preganancies: an ominous finding with adverse pregnancy outcome. Am. J. Obstet. Gynecol., 1988, 159:559.

18. Divon MY, Girz BA, Lieblich R, Langer O. Clinical management of the fetus with markedly diminished umbilical artery end-diastolic flow. Am. J. Obstet. Gynecol., |989, |61: I 523-27.

19. Tyrrell S, Obaid AH, Lilford RJ. Umbilical artery Doppler velocimetry as a predictor of fetal hypoxia and acidosis at birth. Obstet. Gynecol., 1989,74:332-7.

20. Forouzan I. Absence of end-diastolic flow velocity in the umbilical artery: a review. Obstet Gynecol Surv 1995; 50:219-27.

21. Zugaib M, Miyadahira S, Nomura RMY, Francisco, RPV. Vitalidade Fetal. Propedêutica e Avaliação. Capítulo 9. Editora Atheneu. São Paulo 2000.

22. Francisco RPV. Predição da acidose no nascimento em gestações com diástole zero ou reversa à dopplervelocimetria das artérias umbilicais. São Paulo, 1998. p.97. Tese (mestrado) Faculdade de Medicina, Universidade de São Paulo.

23. Battaglia C, Artini PG, Galli PA et al. Absent or reverser end- diastolic flow in umbilical artery and severe intrauterine growth retardation. An ominous association. Acta. Obstet. Gynecol. Scand., 1993,72:167-171.

24. Bell JG, Ludomirsky A, Bottalico J, Weiner S. The effect of improvement of umbilical artery absent end-diastolic velocity on perinatal outcome. Am. J. Obstet. Gynecol, 1992, 167: I0I5-20.

25. Rochelson B. The clinical significance of absent end-diastolic velocity in the umbilical artery waveforms. Clin. Obstet. Gynecol., 1989, 32:692-702.

26. Malcolm G, Ellwood D, Devonald K et al. Absent or reversed end diastolic flow velocity in the umbilical artery and necrotising enterocolitis. Arch. Dis. Child., 1991,66:805-7.

27. Al-Gazali W, Chapman MG, Chita SK et al. Doppler assessment of umbilical artey blood flow for prediction of outcome of fetal cardiac abnormality. Br J Obstet Gynaecol 1987, 94: $742-45$.

28. McCowan LM, Lawrence MB, Erniske A et al. Umbilical artey Doppler blood flow studies in the preterm, small-for-gestational-age fetus. Am J Obstet Gynecol 1987; 156:655-59.

29. Fisk NM, MacLachlan N, Eliss $C$ et al. Absent end-diastolic flow in first trimester umbilical artery Lancet 1988; 2: 1256-57.

30. Peeters LH, Sheldon RE, Jones MD et al.. Blood flow to fetal organs as a function of arterial oxigen content. Am. J. Obstet. Gynecol., 1979, 135:637-46.

31. Kurkinen-Räty M, Kivelä A, Jouppila P. The clinical significance of an absent end-diastolic velocity in the umbilical artery detected before the $34^{\text {th }}$ week of pregnancy. Acta Obstet Gynecol Scand 1997;76:398-404.

32. Weiss E, Ulrich S, Berle P. Blood flow velocity waveforms of the middle cerebral artery and abnormal neurological evaluations in liveborn fetuses with absent or reverse end-diastolic flow velocities of the umbilical arteries. Eur. J. Obstet. Gynecol. d Rep. Biol., 1992. 45:93-100

33. Pattinson RC, Odendaal H J, Kirsten G. The relationship between absent end-diastolic velocities of the umbilical artery and perinatal mortality and morbidity. Early Hum. Dev., 1993,33:61-9.

34. Macparland P, Steel S, Pearce M. The clinical implications of absent or reversed end-diastolic frequencies in umbilical artery flow velocity waveforms. Eur. J. Obstet. Gynecol. 1990,37: 15-23.

35. Weiner CP. The relationship between the umbilical artery systolic/diastolic ratio and umbilical blood gases measurements in specimens obtained by cordocentesis. Am. J. Obstet. Gynecol., 1990, 162:1 198-202.

36. Steinman D, Miyadahira S, Yamamoto RM, Francisco RPV, Zugaib M. Estudo do $\mathrm{pH}$ do sangue da artéria umbilical em 61 fetos com diagnóstico de diástole zero ou reversa na dopplervelocimetria da artéria umbilical Rev. Bras. Ginec. Obstet., 1997, 19:50.

37. Miyadahira S, Yamamoto RM, Francisco RPV, Steinman DS, Zugaib M. Avaliação da vitalidade fetal em I 43 casos de diástole zero ou diástole reversa na dopplervelocimetria da artéria umbilical. Rev. Bras. Ginec. Obstet. | 997, 19:51.

38. Yamamoto RM, Miyadahira S, Francisco RPV, Steinman D, Zugaib, M. Resultados perinatais em gestantes com história de natimorto em gestação anterior e diástole zero na dopplervelocimetria da artéria umbilical. Rev. Bras. Ginec. Obst., 1997, 19:51.

39. Lopes LM, Kahhale S, Zugaib M. Diástole-zero da artéria umbilical: correlação da sua duração com o prognóstico fetal. Rev. Ginec. Obstet. 1990, I:68-72.

40. Woo JSK, Liang ST, Lo RLS. Significance of an absent or reversed end diastolic flow in Doppler umbilical artery waveforms. J. Ultrasound Med., 1987,6:291-7.

41. Hsieh F, Chang F, Ko T, Chen H, Chen Y. Umbilical artery flow velocity waveforms in fetuses dying with congenital anomalies. Br. J. Obstet. Gynaecol., 1988,95:478-82.

42. Schulman H. The clinical implications of Doppler ultrasound analysis of the uterine and umbilical arteries. Am. J. Obstet. Gynecol. 1987, 1 56:889-93.

43. Bekedam DJ, Visser GHA, Van Der Zee AGJ, Snijders RJ, Poelmann-Weesjes G. Abnorma velocity waveforms of the umbilical artery in growth retarded fetuses: relashionship to antepartum late heart rate decelerations and outcome. Early Hum. Dev., 1990,24:79-89.

Artigo recebido: 18/12/2000 Aceito para publicação: 24/0 I/200 I 